Fecha de recepción: 09-10-2017

Fecha de aceptación: 24-01-2018

Link para este artículo: http://dx.doi.org/10.14198/ALEUA.2018.29-30.09

Puede citar este artículo como:

TRECCA, Simone, «Juventudes universitarias y teatro. Apuntes para un estudio comparativo de la primera etapa del teatro español universitario y de los Teatri G.U.F.», Anales de Literatura Española, n. ${ }^{\circ}$ 29-30 (2018), pp. 191-203.

\title{
JUVENTUDES UNIVERSITARIAS Y TEATRO. APUNTES PARA UN ESTUDIO COMPARATIVO DE LA PRIMERA ETAPA DEL TEATRO ESPAÑOL UNIVERSITARIO Y DE LOS TEATRI G.U.F.
}

\author{
SIMONE TRECCA \\ Università degli Studi Roma Tre
}

Las reflexiones expuestas en el presente trabajo se fundamentan en algunas observaciones previas de carácter histórico y político y a estas alturas no pretenden tener más alcance que el de ir apuntando posibles líneas de investigación y motivos de profundización en el estudio de las primerísimas andaduras del teatro español universitario. Representan, de hecho, el intento de proponer una aproximación a dicho fenómeno desde la perspectiva de su colocación en el contexto internacional del surgimiento y desarrollo de los fascismos europeos y, básicamente, del modelo italiano, teniendo en cuenta las relaciones concretas (así como las afinidades y divergencias menos directas) existentes entre los sistemas objeto de comparación.

Hace tiempo Miguel Ángel Ruiz Carnicer señaló oportunamente que las organizaciones estudiantiles de raigambre fascista surgieron, tanto en España como en Italia y Alemania, «con anterioridad a la toma del poder y, por lo tanto, con una vocación de lucha política en un ambiente hostil, lo que les otorga una serie de rasgos comunes» (1991: 66). En lo referente a los dos países que aquí nos ocupan, la trayectoria inicial de ambas corporaciones se ve afectada por mecanismos muy parecidos de búsqueda de un equilibrio entre las instancias de las juventudes fascistas y las necesidades de los partidos a los que ellas se sometieron formalmente (el Partito Nazionale Fascista y Falange Española, respectivamente), que querían aprovechar el ímpetu de las nuevas generaciones universitarias y, al mismo tiempo, contener y controlar tendencias centrífugas a través de la doctrina y la propaganda organizada. También fue rasgo común 
al comienzo el uso de la violencia escuadrista para con los representantes de las fuerzas antifascistas y, por otro lado, la contraposición con las agrupaciones estudiantiles católicas, ${ }^{1}$ coherentemente con el afán de fascistización que caracteriza, como es obvio, las fases fundacionales y de asentamiento de dichos movimientos. Finalmente, aspecto que constituye la repercusión más interesante para los fines del presente trabajo y de las investigaciones comparativas que en él propongo, tanto los Gruppi Universitari Fascisti (GUF) como el Sindicato Español Universitario (SEU) hicieron hincapié desde el principio en la organización y el control de varias actividades culturales, como instrumentos revolucionarios y de propaganda, sirviendo los primeros como modelo en muchos campos, entre ellos la prensa universitaria y el teatro experimental. ${ }^{2}$

Además de tales semejanzas, es oportuno recordar, sin embargo, que el nacimiento de los GUF entre 1919 y 1920 se adelanta casi tres lustros al del SEU, cuya institución prácticamente coincide con la oficialización de Falange Española en 1933. Se trata de un lapso de tiempo muy amplio, en vista sobre todo del clima político y social convulso que lo caracteriza, durante el cual las relaciones entre Italia y España fueron muy intensas, tanto a nivel oficial como desde el punto de vista de las influencias de la primera en la evolución del fascismo español, lo que repercute especialmente en la organización del sector universitario y de la educación, así como en las políticas culturales. ${ }^{3}$ Es muy cierto, en efecto, que « [a] partir de la Guerra Civil y en la inmediata posguerra, los líderes del SEU se desplazarán a Italia para conocer el funcionamiento de los GUF» (Ruiz Carnicer, 1991: 90), así como que dicha correspondencia se manifiesta de manera bastante significativa también en el más estricto ámbito del teatro entre finales de los treinta y principios de los cuarenta, como veremos más adelante. Pero no hay que olvidar que, en general, el interés de Mussolini

1. Como apunta La Rovere (2003: 159-172), los GUF, después de una época de duros contrastes, también marcados por actos de violencia escuadrista, consiguen aislar en 1931 a los universitarios católicos agrupados en la FUCI (Federazione Universitaria Cattolica Italiana) y obtener el monopolio. Termina así la labor de conquista de las universidades empezada ya a mediados de los 20.

2. Ruiz Carnicer (1991: 85, 89-90) subraya que las actividades culturales no fueron tan relevantes como estrategia política en el caso de la organización estudiantil de la Alemania nazi y, además, de esta no puede afirmarse que se inspiró en los GUF como, en cambio, lo hizo muy patentemente el SEU. Sobre la prensa del SEU (con algunas menciones del modelo del periodismo estudiantil italiano, léase Ruiz Carnicer, 1996b). Del mismo autor, sigue siendo una referencia imprescindible para el estudio del SEU (y, por consiguiente, de las bases ideológicas de los TEUs de la primera época) el libro que consagra al análisis de la evolución de la organización desde el año 1939 hasta 1965 (Ruiz Carnicer, 1996a).

3. «El fascismo italiano siempre fue para el SEU un modelo mucho más cercano que el del nazismo alemán, a pesar del también numeroso intercambio de visitas y puntos de vista con Berlín» (Ruiz Carnicer, 1991: 90). 
(y de los intelectuales del fascismo) por la evolución de la situación política española se remontaba a la década de los veinte y se había ido concretando en un programa de acciones (que también fue transformándose con el tiempo) dirigidas a influir enormemente en dicho proceso. Se trató, en palabras de Dimas Vaquero, de un verdadero «acoso cultural y propagandístico iniciado ya en la dictadura de Primo de Rivera, con algunos obstáculos y dificultades durante la II República, pero que culminaría con gran éxito tras la llegada de los legionarios italianos y su participación en la Guerra Civil» (2017: 117) y se articuló desde el principio con el fin explícito de abarcar la mayoría de los ámbitos sociales, entre los cuales destacaban, desde luego, la educación y la prensa. En lo que atañe a nuestro objeto de análisis, se programaban viajes de estudio de universitarios españoles a Italia ya antes del surgimiento del SEU y de la época de las visitas oficiales de los directivos de las organizaciones juveniles. De hecho, todo quedaba dentro de un claro proyecto de difusión del fascismo en España que, de alguna manera, puede leerse como un intento de colonización cultural, por parte del duce y de los políticos e intelectuales en el poder durante el ventennio. ${ }^{4}$ Por otra parte, son muy conocidas las relaciones directas entre los ideólogos falangistas y el fascismo italiano, ya desde ese primer viaje iniciático de José Antonio Primo de Rivera, cuando en 1923, a sus veinte años, acompañó a su padre a Roma durante una visita oficial de él y de Alfonso XIII, y luego por su cuenta durante la época de la II República, en 1933 y 1935, cuando viajó con el fin de obtener apoyo político y subvenciones para sí y para su movimiento.

Es evidente, pues, que los intercambios entre los llamados «jóvenes con inquietudes» de ambos países se trabaron ya desde los primerísimos años del fascismo, en el marco de la política de expansión del duce y de la necesidad de legitimación de Falange, y fueron consolidándose al reforzar su carácter de propaganda y su virulencia durante los años de la Guerra Civil, hasta institucionalizarse en la inmediata posguerra. Pese a que la intervención italiana en la guerra di Spagna no llegara a tener el eco mediático que había merecido anteriormente la campaña de Mussolini en Etiopía, es indudable que los GUF interpretaron su participación militar como «una nuova battaglia per la grandezza del fascismo, la sfida alle diplomazie occidentali e soprattutto la lotta al bolscevismo» (Duranti, 2008: 276). ${ }^{5}$ Reverdeció así el mito del volontarismo

4. Acúdase, al respecto, al indispensable libro de Victoriano Peña Sánchez (1995). Sobre la actitud de Mussolini ante la II República española, léase Saz (1986).

5. Simone Duranti dedica una parte importante de su volumen consagrado a la actividad de los GUF entre 1930 y 1940 a la llamada en Italia guerra di Spagna, sobre todo a través de su repercusión en la prensa universitaria de aquellos años (2008: 276-307). 
goliardico que había caracterizado los movimientos estudiantiles italianos desde sus inicios, también gracias a la propaganda contra la amenaza roja ocasionada por la cruzada de los nacionales en España. Una vez terminada la contienda y con Franco en el poder, ${ }^{6}$ las relaciones culturales se hacen más y más oficiales, con la institución, por ejemplo, del Istituto Italiano di Cultura en Madrid, a finales de 1939, bajo la dirección primero de Salvatore Battaglia (hasta 1941) y, luego, de Ettore de Zuani hasta el final de la II Guerra Mundial. Este, con respecto al primero, fue un hombre más identificado con las posiciones culturales del régimen y seguramente más apto para seguir con la política de difusión de los mismos en España y empezar una campaña de propaganda de la cultura española como espejo de los nuevos valores exportados: suyos son dos artículos sobre la «rivoluzione spagnola» publicados en la revista Nuova Antologia justo al comienzo de la Guerra Civil (Zuani, 1936a; 1936b), así como otros escritos como corresponsal en el extranjero, aparecidos en la revista Legioni e Falangi. Rivista d'Italia e di Spagna, a principios de los veinte. ${ }^{7}$ No sorprende por lo tanto que, nada más obtener el encargo al frente del Istituto, se le otorgasen con frecuencia una o dos páginas de la revista Scenario para la publicación de noticias y críticas de puestas en escena principalmente de la cartelera madrileña. El director del periódico, Nicola de Pirro, ${ }^{8}$ no había tenido el mismo detalle con Battaglia, aunque sí se había publicado durante el encargo de este un artículo sobre teatro español, firmado por Raffaele Mastrostefano, a propósito de la «Grandezza di Lope de Vega» (1940), que comentaremos más adelante.

En lo referente a los sectores estudiantiles y a su compromiso en dichas políticas de intercambio cultural, y específicamente teatral, es muy oportuno mencionar el acto que el TEU de Sevilla celebró en honor precisamente de Ettore de Zuani, el 16 de noviembre de 1941, con la puesta en escena de La posadera de Goldoni: todo un estreno si se considera que, en la misma temporada, el clásico italiano se repuso en el Teatro Lope de Vega en otra ocasión aún más oficial, el 5 de julio de 1942, esto es, durante la visita a la capital andaluza

6. Sobre las relaciones difíciles entre Mussolini y Franco, véase la reciente contribución de Vaquero (2017).

7. Se trata de una publicación mensual, que salió entre el mes de octubre de 1940 y el de julio de 1943, tanto en edición italiana como española (con el título Legiones y Falanges). Sobre esta revista, véase Sinatra (ed.), 2015.

8. Nicola de Pirro fue Ispettore del Teatro y luego, desde 1942, presidente del Ente Teatrale Italiano (E.T.I.) recién fundado por voluntad del ministro del Ministerio de Cultura Popular (el llamado Minculpop), el gerarca Alessandro Pavolini. La revista Scenario, que siempre otorga amplio espacio a los temas del teatro juvenil y universitario, resulta un documento muy útil para el estudio de este fenómeno en la Italia fascista. 
nada menos que de Giorgio Venturini, jefe nacional de los Teatri-GUF y Vieri Bigazzi, a la sazón director del Teatro-GUF de Florencia. ${ }^{9}$ La estancia en España de la delegación del teatro universitario italiano tuvo su repercusión también en la prensa nacional, como puede verse, por ejemplo, en un suelto del $A B C$ del 7 de julio, en el cual se mencionan tanto las identidades y los cargos de los invitados (a pesar de los errores en la referencia a Bigazzi) como la naturaleza del acto teatral (también con imprecisiones), que contempló la representación, además del texto goldoniano, de unas escenas de La vida es sueño y el primer acto del Fausto de Goethe. Como señala oportunamente María Jesús Bajo Martínez, quien recogió hace tiempo la noticia, «fiel a la ideología falangista, el TEU de Sevilla mantiene contactos con los grupos universitarios fascistas» (1999: 224), lo cual confirma que en los entornos culturales directamente controlados e influidos por Falange y, en el ámbito universitario, por el SEU, las relaciones con los ambientes fascistas italianos representan un rasgo esencial.

También en este caso hay que poner de relieve que no se trató ni de un evento aislado ni del primer punto de contacto oficial, ya que existen unos antecedentes que, a mi manera de ver, pueden ofrecer una lectura bastante clara de la naturaleza de la visita de 1942. En primer lugar, el Teatro Sperimentale dei GUF de Florencia había puesto en escena, el 6 de junio de 1938, la traducción y adaptación italiana de El alcalde de Zalamea de Calderón firmada por Raffaello Melani, que por aquel entonces estaba al mando de la Scuola di recitazione del Teatro, una escuela de actuación que representó, en su momento, un lugar de referencia para la renovación del arte escénico italiano (Giachetti, 1939). ${ }^{10}$ Tanto la noticia como el texto integral de la versión se acogieron en las páginas de la revista Scenario (Calderón de la Barca, 1938), ${ }^{11}$ en plena Guerra Civil, es decir cuando los jóvenes universitarios italianos, como hemos visto, se apuntaban voluntarios a la cruzada y al mismo tiempo que en Sevilla y Granada, pertenecientes a la zona sublevada, se hacía ya muchísimo teatro, sobre todo como forma asistencial. ${ }^{12}$ En segundo lugar, el ya mencionado artículo de 1940 de Mastrostefano, en el que se ensalza la figura de Lope de Vega representa, sin duda, un acto muy consciente de consagración cultural fomentado por el

9. Ambos eventos se mencionan en el n. 4 (extraordinario) del folleto Teatro, publicado por el TEU de Sevilla. Le agradezco enormemente a Antonio Serrano el envío de este material.

10. Sin olvidar, desde luego, la Accademia d'Arte Drammatica fundada por Silvio d'Amico en 1936, o la realidad del Teatro delle Arti de Anton Giulio Bragaglia.

11. Esta interesante operación de importación del canon teatral español merece sin duda un estudio que, por su naturaleza y por motivos de espacio, tengo que dejar para otra publicación.

12. Lo subrayó Bajo Martínez durante el seminario y compartió conmigo en varias conversaciones noticias y consideraciones al respecto.

Anales, 29-30 (2018), pp. 191-203 
reconocimiento del peso de «l'opera del Fascismo per il trionfo delle forze sane della sorella Nazione spagnola» y, de alguna manera, ofrece una semblanza del Fénix de los ingenios como poeta de la patria, con una finalidad de propaganda y apropiación ideológica patente. No faltan, sin embargo, apreciaciones estéticas de cierto relieve, muy acordes con el clima de renovación del teatro que había caracterizado en Italia, con varias fases, la década de los treinta y los comienzos de los cuarenta, como veremos más adelante. ${ }^{13}$ Imposible, asimismo, no comentar la proximidad de la fecha de publicación de estas dos páginas de homenaje con la visita de los altos mandos del SEU a Bolonia, a finales de abril del mismo año, durante la celebración de la que habría de ser la última edición de los Littoriali della Cultura e dell'Arte. ${ }^{14}$ Viajaron a Italia personalidades de peso como Rafael García Serrano, Manuel Pardo Suárez y, sobre todo, Fernando de Alzaga y Rubio, por aquel entonces al mando de la Delegación Nacional de Prensa y Propaganda y de la revista Haz. La prensa nacional italiana, por supuesto, difunde la noticia con las acostumbradas estrategias de apropiación y con el fin de subrayar el valor del modelo fascista: un artículo de Il Corriere della sera fechado 30 de abril, por ejemplo, se titula «I Littoriali della cultura. Un ricevimento in onore della Delegazione goliardica spagnola» (con un uso muy sutil del término nacional goliardica) y, sobre todo, pone de relieve el hecho de que la «Delegazione spagnola [...] ha visitato minutamente il Comando dei Littoriali, interessandosi al funzionamento dei singoli uffici e compiacendosi per l'ottima organizzazione», esto es, que ha llegado a Italia para aprender cómo se programa la cultura en un estado fascista.

A todo ello hay que añadir otras circunstancias más que preparan y acompañan, a mi modo de ver, la visita de la delegación italiana de 1942, a saber: el encargo a Enrico Fulchignoni de la puesta en escena de La Celestina en el Teatro

13. Léanse, por ejemplo, las siguientes palabras: «Rinnovare il teatro senza interrompere bruscamente la tradizione, anzi conservando e potenziando quanto, in essa, vi era di tipicamente spagnolo e, insieme, capace di essere investito, vivificato e universalizzato dal soffio dell'arte: questa la sostanza - di valore perenne- dell'opera di Lope de Vega» (Mastrostefano, 1940: 230).

14. Los Littoriali della cultura e dell'arte fueron una manifestación cultural con carácter competitivo que, en palabras de La Rovere, representaron «l'arena intellettuale che il regime mise a disposizione dei giovani per discutere, sotto il controllo del partito, i principali problemi del tempo fascista. Essi costituivano parte integrante dell'apparato educativo creato dal Pnf per attuare il progetto di formazione e mobilitazione permanente delle giovani generazioni» (2003: 265). A la primera edición de Florencia, en 1934, siguieron la de Roma al año siguiente y las de Venecia, Nápoles, Palermo, Trieste y Bolonia hasta 1940, cuando se interrumpió la organización del evento por el ingreso de Italia en la segunda guerra mundial. Para una valoración de los Littoriali como expresión de la juventud fascista, véanse La Rovere (2003: 265-289) y Alfassio Grimaldi, Addis Saba (1983). 
delle Arti de Roma, en la temporada 1940-1941; el montaje de El ayunador (Il digiunatore), del mismo Fulchignoni, que realizó el TEU en el Teatro Español el 6 de mayo de 1942; ${ }^{15}$ la representación por parte del Teatro Nazionale del GUF de Florencia de Unificación, de Jacinto Miquelarena, en el curso 1941-1942. ${ }^{16}$ Para valorar el peso de dichos acontecimientos, es oportuno recordar aquí algunos elementos útiles y encuadrarlos en su contexto, empezando por ofrecer unos detalles sobre la figura de Fulchignoni y su envergadura en el ámbito del teatro universitario y juvenil italiano. ${ }^{17}$ Nacido en 1913, este innovador director de escena siciliano figura entre los ganadores de los certámenes de crítica teatral en dos ediciones de los Littoriali della Cultura e dell'Arte (en Venecia, 1936 y Palermo, 1938) y estuvo al frente del Teatro GUF de Messina desde sus comienzos en 1936 y hasta 1939. Fue entre los mayores impulsores de la renovación del arte escénico italiano, como puede desprenderse ya al leer su comentario sobre la andadura del teatro universitario que dirigió en su ciudad: «la necessità della creazione di un Teatro Universitario si cercò di postularla in una sfera di volori ideali, dai quali spontanea nascesse una gustificazione dell'iniziativa: l'opportunità di riesumare alcune grandi opere del passato, di rinnovare attraverso la realizzazione scenica conoscenze letterarie e scolastiche, di suscitare infine una corrente di idee intorno al dramma moderno» (Fulchignoni, 1938: 458); más adelante, recuerda que «nel G.U.F. esiste la sezione che si occupa del repertorio moderno, e che costituisce il vero e proprio "Sperimentale"» entre cuyas premisas destaca la «necessità della messa in scena di opere brevi ma significative di autori atti a impersonare le tendenze del dramma contemporaneo, dall'inizio del nostro secolo a oggi» (459). Incansable defensor, pues, de un difícil pero necesario equilibrio entre la conservación de todo un patrimonio

15. Saco la noticia de la tesis de Serigne Mahanta Kébé (1994: 353-355), agradeciéndole a Javier Huerta el envío de esta útil referencia bibliográfica. Il digiunatore se había montado en el Teatro dell'Università di Roma en 1941 (no he llegado a localizar la fecha exacta de la representación).

16. Me enteré de ello a través del mencionado folleto publicado por el TEU de Sevilla (1942), en el que se publica una foto de la puesta en escena con la acotación siguiente: «Los camaradas A. Geri y E. Borri en una escena de Unificación, de Jacinto Miquelarena, representada por el Teatro Nacional del GUF de Florencia, bajo la dirección y escenografia de Vieri Bigazzi». No he llegado hasta el momento a localizar otras fuentes con indicación de la fecha de representación.

17. Mencionemos asimismo, si bien al margen de estas reflexiones, el hecho de que Fulchignoni fue entre los directores de escena directamente subvencionados por el Minculpop (con 12.000 liras totales), según se aprende de la documentación inherente a las financiaciones de dicha administración durante la década 1933-1943 (sobre el uso de las subvenciones del teatro durante la época fascista, véase Scarpellini, 1989: 195-203). Junto a su nombre aparecen el de Anton Giulio Bragaglia, director del Teatro delle Arti (con 46.000 liras) y Giorgio Venturini (con 6.000 liras de ayudas).

Anales, 29-30 (2018), pp. 191-203 
teatral (se refiere exclusivamente en sus escritos de aquellos años a la tradición popular) y la urgencia de renovación, mantuvo una atención constante hacia la actividad y el papel de los jóvenes para alcanzar dichos objetivos, sobre todo desde la perspectiva de la lucha contra la tradición del teatro burgués, como manifestó en varias ocasiones, entre otras en una crónica del Convegno Nazionale dei GUF de 1942 que publicó en la revista Scenario: «La intransigenza che i giovani di oggi manifestano verso le forme svilite e corrotte che altre civiltà, come quella borghese, ci hanno lasciato in tenace quanto deprecabile retaggio, sono la garanzia più sicura per una sempre più decisa impostazione, in senso italiano, del problema del teatro» (Fulchignoni, 1942: 51). Se trata de consideraciones que se insertan en el marco de una agria polémica sobre el rol de giovani e vecchi en el teatro, que se había venido desarrollando entre finales de los treinta y principios de los cuarenta, y de la cual él mismo había sido en parte protagonista. Especialmente, a raíz de su controvertido montaje de Nuestra ciudad de Thornton Wilder, en el Nuovo Teatro de Milán el 28 de marzo de 1940, después de su puesta en escena en el Teatro delle Arti de Roma, por voluntad del otro gran renovador de la escena que fue Bragaglia. La falta de acción dramática y de conflicto que caracteriza la obra, acompañada de una escenificación mínima, sin ningún objeto sino los que los actores fingían con sus gestos y movimientos, representaron elementos tan revolucionarios como para producir una reacción violenta del público más conservador que terminó en una verdadera riña. ${ }^{18} \mathrm{~A}$ las inquietudes de los representantes más jóvenes de la vida teatral, el régimen respondía muy cautelosamente, sobre todo porque no podía renunciar al apoyo de esa franja de la sociedad ya con un pie en la II Guerra Mundial y, en efecto, subvencionó varios proyectos y permitió que, a pesar de la interrupción de los Littoriali, se celebrase en Génova, en 1942, el ya mencionado congreso nacional de los GUF, sin que ello supusiera una distensión de los mecanismos de control ejercidos por el Partido Nacional Fascista (Scarpellini, 1989: 300-306). ${ }^{19}$

En vista de todo lo dicho, no sorprende que el ambiente innovador del TEU se interese por una figura como la de Fulchignoni y que, de alguna manera,

18. Eleonora Scarpellini (1989: 301-303) menciona que asistieron al espectáculo, entre otros, unos conmovidos Paolo Grassi y Giorgio Strehler, que recordarían el evento como un hito en la historia del llamado en Italia teatro di regia.

19. El apoyo del régimen se manifestó, asimismo, con mecanismos de promoción institucional de los jóvenes. Menciono solamente, como ejemplo pertinente al tratarse del ámbito teatral y de una de las personalidades que viajaron a España en la comentada visita de 1942, el hecho de que Giorgio Venturini fue nombrado Direttore Generale dello Spettacolo en la época de la Repubblica di Salò. 
se haga hincapié en su papel como joven director de escena vinculado con los ámbitos universitarios para establecer un nexo entre las actividades teatrales de las dos naciones fascistas del Mediterráneo. Ni es de extrañar que desde España se asemeje su talante al del invitado oficial de 1942, Giorgio Venturini, también director de escena, si se considera la posición institucional de este (al frente del Teatro Sperimentale GUF de Florencia y, luego, del Teatro Nazionale GUF), así como su actitud crítica y su importante papel en el panorama del teatro de las juventudes universitarias en Italia. Baste con leer las consideraciones que a continuación propongo, sacadas de un artículo cuyo llamativo título, «Teatro di domani», pone ya de manifiesto la mirada puesta en el rol de los jóvenes para el desarrollo futuro de la escena italiana y la plena realización de lo que, al fin y al cabo, fue el ideal de regeneración teatral de toda una generación:

Esiste in Italia un repertorio mai rappresentato o raramente rappresentato. un repertorio ritrovato, a poco a poco, dalle appassionate fatiche dello Sperimentale di Firenze, dal fervore di tutti i Teatri G.U.F. e d'eccezione d'Italia, dalle polemiche di molti periodici. Un repertorio il quale, in ugual misura di quello normale, ha il diritto di essere conosciuto, perché potrebbe contenere gli elementi per la nascita dello spettacolo. La nascita del nuovo spettacolo, forse: che domani popoli le platee, in virtù di un interesse artistico e sociale. Allora si vedrà quanto sia stata proficua l'esperienza fatta nei piccoli teatri e la preparazione perseguita polemicamente attraverso la ricerca di nuovi indirizzi nel quadro del teatro contemporaneo italiano (Venturini, 1942: 405).

Finalmente, la puesta en escena del breve texto de Miquelarena por el Teatro GUF de Florencia, bajo la dirección del otro delegado que visitó España en 1942, Vieri Bigazzi, además de confirmar la existencia de un mecanismo de reconocimiento mutuo, pone de relieve, en mi opinión, la diferente situación de las dos realidades: comprometido, el uno, en una nueva guerra y, de hecho, en la defensa del fascismo a escala mundial; empeñado, el otro, en consolidar el nuevo régimen y preservar las posiciones ideológicas de Falange. La recuperación de la obrita del autor bilbaíno, publicada originalmente como artículo dialogado titulado Por España, unidos en la guerra y en la muerte, que había obtenido el premio Mariano de Cavia de 1937, es muy representativa del clima bélico al que desde el GUF de Italia se quiere apuntar y, por otra parte, manifiesta la intención de referirse a la otra guerra recientemente combatida, también por los universitarios italianos, en defensa del fascismo. ${ }^{20}$

En consideración de todo lo que antecede, me parece evidente que al intercambio institucional oficial entre el ámbito estudiantil español y el italiano, se

20. No excluyo que se tratara también de un intento de persuasión, frente a la neutralidad de España.

Anales, 29-30 (2018), pp. 191-203 
sumaron esfuerzos mutuos por establecer puntos de contacto, no solamente formales, entre dos realidades que atravesaban períodos muy distintos de sus trayectorias. Es indudable que la visita de los delegados del SEU a Bolonia, en 1940, representó una especie de estancia de aprendizaje con el fin de estudiar el modelo de los Littoriali y la estructura que el fascismo quiso dar al sector cultural nacional y no creo que sea inoportuno interpretar la de los jefes de los teatros universitarios italianos dos años después también como un examen, en el que la organización estudiantil más joven quiso dar muestra de lo aprendido ${ }^{21}$ incluso en términos de repertorio, dada la elección de Goldoni, que las políticas culturales del fascismo estuvieron consagrando, en efecto, en aquellos años hasta programar uno de sus textos en la Exposición Universal de Roma de 1942, que nunca llegó a celebrarse. Pero también es incuestionable que los dos sistemas que intentaron dialogar a la sazón se encontraban en situaciones muy dispares: prueba de ello es, ya a nivel oficial, el hecho de que los representantes italianos que viajaron pertenecían estrictamente al ámbito del teatro universitario y no, como en el caso de los delegados españoles, a las jerarquías de la corporación o del partido en un sentido más amplio. Los primeros gozaban sin duda de una cierta autonomía, perteneciendo a una realidad muy consolidada, y podían representar directamente, como figuras institucionales, el régimen y el partido, sin que fuese necesaria ninguna mediación y, al mismo tiempo, funcionar como legitimadores, en España, de las buenas prácticas del movimiento falangista y de su sindicato. Si nos fijamos, finalmente, en el tipo de operaciones de promoción y producción (en las tablas como en las páginas de la prensa) que marcaron el intercambio cultural durante los pocos años que acabamos de analizar, resulta bastante claro que, si por un lado se tiende a confirmar mutuamente el peso de los clásicos en la constitución del repertorio de los respectivos Estados, por otro se asume el papel que ambos sistemas culturales quieren programáticamente otorgar a los jóvenes autores, directores, escenógrafos, bien en su calidad de creadores originales bien como innovadores capaces de transmitir a la civiltà nuova los valores patrióticos, ideales, míticos, hasta diría fundacionales, que se atribuyen a ese mismo patrimonio clásico.

Los pocos ejemplos que he venido comentando en las breves páginas de esta primera aproximación al estudio comparativo propuesto nos brindaron la ocasión de interpretar algunos de los aspectos del contexto político y cultural

21. Como apunta César Oliva, el SEU «retomó en los años cuarenta los trabajos teatrales en facultades y escuelas. Empezó por dar carácter competitivo a las muestras salidas de cada distrito universitario, lo cual ofreció ciertos estímulos en la producción escénica. [...] De esta manera, el SEU vehiculaba las inquietudes estudiantiles, neutralizando cualquier posibilidad crítica que el teatro pudiera adoptar» (1999: 16-17). 
de las posibles relaciones entre los planteamientos del primer teatro universitario español (con y sin la mediación directa de las jerarquías del SEU) y el modelo italiano de los Teatri-GUF. Son muchas las consideraciones y las referencias que dejo en el tintero, no por ser inoportunas o accesorias, sino porque nos llevarían ya a perseguir las líneas de investigación que aquí, en cambio, solamente pretendo sugerir; de hecho me limito, para concluir, y en atención a lo expuesto, a indicar como posibles perspectivas de estudio algunos temas y objetivos que me parecen especialmente relevantes y viables. En primer lugar, resultaría muy conveniente profundizar en el análisis comparativo del papel de la juventud universitaria en los respectivos sistemas políticos e ideológicos nacionales, así como la evolución de sus relaciones con el partido del que dependen. De especial interés, como base para su aplicación en contextos más estrictamente culturales y teatrales, sería estudiar el posicionamento de las corporaciones estudiantiles y, luego, de las figuras más representativas de ambas, ante algunos temas típicos como la mitificación de la juventud y de lo nuevo y la necesidad de un conflicto generacional; la adhesión a las políticas de masas; las maneras de actuar del proceso de fascistización del sector de la educación, la cultura, la prensa, etc.; las declinaciones del concepto de propaganda y su aplicación al ámbito artístico. Desde una perspectiva más rigurosamente inherente al desarrollo de los teatros universitarios y a la interpretación de su rol, por un lado dentro de dichos programas políticos e ideológicos, por otro en su relación con el arte escénico nacional de referencia, ya una mirada parcial y superficial a los acontecimientos, escritos, opiniones y producciones de esa época hace que destaquen unos rasgos comunes que merecen, sin duda, un estudio más pormenorizado: la reacción ante una dramaturgia aburguesada o de evasion; el rechazo del teatro como puro entretenimiento, aun sin renunciar a los aspectos más espectaculares y grandiosos; el afán de experimentación formal y técnica; la preocupación por el repertorio y la función clave de los jóvenes de cara a la realización de un proceso de renovación a partir de la tradición. Una lectura atenta de las intervenciones de los protagonistas del teatro universitario italiano y español (artículos de prensa, críticas, textos de sus conferencias, obras teatrales, en su caso, críticas sobre sus realizaciones escénicas como directores, escenógrafos, etc.), con el fin de confrontarlas sistemáticamente en busca de elementos de continuidad o discontinuidad, de afinidades o afiliaciones explícitas, etc., puede dar lugar, en mi opinión, a una serie de hallazgos muy notables para contribuir a una interpretación de las primeras andaduras del TEU y, posiblemente, para ir apuntando, gracias a la comparación, también actitudes diferenciadas de los varios TEUs locales. 


\section{Bibliografía citada}

Alfassio GRIMAldi, Ugoberto, Marina AdDIS SABA, Cultura a passo romano. Storia e strategie dei Littoriali della cultura e dell'arte, Milano, Feltrinelli, 1983.

BAjo MARTíneZ, María Jesús, «Tres décadas del teatro universitario en Sevilla», en L. García Lorenzo (ed.), Aproximación al teatro español universitario (TEU), Madrid, CSIC, 1999, pp. 223-233.

Calderón de la BarCa, Pedro, L'alcàde di Zalamea, versión de Raffaello Melani, Scenario, ottobre 1938, pp. 541-556.

DURANTI, Simone, Lo spirito gregario. I gruppi universitari fascisti tra politica e propaganda (1930-1940), Roma, Donzelli, 2008.

FUlCHIGNONI, Enrico, «Lo sperimentale del G.U.F. di Messina», Scenario, settembre 1938, pp. 458-461.

FulChignOni, Enrico, «I giovani e il teatro. Il Convegno Nazionale dei G.U.F.», Scenario, febbraio 1942, pp. 50-53.

Giachetti, Cipriano, «Cinque anni di Sperimentale», Scenario, maggio 1939, pp. 233-234.

LA ROVERE, Luca, «Fascist Groups in Italian Universities: An Organization at the Service of the Totalitarian State», Journal of Contemporary History, 34 (3), 1999, pp. 457-475.

-, Storia dei GUF. Organizzazione, politica e miti della gioventù universitaria fascista (1919-1943), Torino, Bollati Boringhieri, 2003.

MAHANTA KÉBÉ, Serigne, Crítica teatral de posguerra en el periódico madrileño "Arriba", tesis doctoral dirigida por Andrés Amorós, Universidad Complutense de Madrid, 1994.

Mastrostefano, Raffaele, «Grandezza di Lope de Vega», Scenario, maggio 1940, pp. 230-231.

Oliva, César, «La escena universitaria española», en L. García Lorenzo (ed.), Aproximación al teatro español universitario (TEU), Madrid, CSIC, 1999, pp. 15-30.

PEÑA SÁNCHEZ, Victoriano, Intelectuales y fascismo, la cultura italiana del ventennio fascista y su repercusión en España, Granada, Universidad, 1995.

RUIZ CARNICER, Miguel Ángel, "Juventud universitaria y fascismo. GUF, NSDStB y SEU. Un análisis comparativo», en Juan José Carreras Ares, Miguel Ángel Ruiz Carnicer (eds.), La universidad española bajo el régimen de Franco, Zaragoza, Institución Ferdinando el Católico, 1991, pp. 63-92.

-, El Sindicato Español Universitario (SEU), 1939-1965. La socialización política de la juventud universitaria en el franquismo, Madrid, Siglo XXI de España, 1996a.

—, «La voz de la juventud. Prensa universitaria del SEU en el franquismo», Bulletin Hispanique, 98 (1), 1996b, pp. 175-199. 
SAZ, Ismael, Mussolini contra la II República. Hostilidad, conspiraciones, intervención (1931-1936), Valencia, Edicions Alfons el Magnánim, 1986.

SCARPELLINI, Emanuela, Organizzazione teatrale e politica del teatro nell'Italia fascista, Firenze, La Nuova Italia, 1989.

SinATRA, Chiara (Ed.), Stampa e regimi. Studi su "Legioni e Falangi / Legiones y Falanges", una "Rivista d'Italia e di Spagna, Bern, Peter Lang, 2015.

TEU DE SEVILla, Teatro, n. extraordinario 4, 1942.

VAqUero, Dimas, Mussolini E España, Franco \& Mussolini. Unas relaciones difíciles, Zaragoza, Comuniter, 2017.

VENTURINI, Giorgio, «Teatro di domani», Scenario, novembre 1942, pp. 405-406.

ZUANI, Ettore de, «Panorama della rivoluzione spagnola», Nuova Antologia, 1547, 1 settembre 1936 .

—, «Le giornate della rivoluzione spagnola», Nuova Antologia, 1548, 16 settembre 1936b. 